\title{
LA INSTRUMENTALIZACIÓN DE LOS NIÑOS Y NIÑAS EN EL CONFLICTO ARMADO COLOMBIANO: DÉCADAS DE LOS 70, 80 y 90
}

\section{The instrumentalization of children in the Colombian armed conflict: decades of the 705,805 and $90 \mathrm{~s}$}

\author{
Sandra Milena Ochoa Pérez \\ sandra.ochoa01@uptc.edu.co \\ Paola Andrea Ochoa Pérez \\ paola.ochoa03@uptc.edu.co
}

\author{
Universidad Pedagógica Tecnológica de Colombia \\ Universidad Formación de Educadores e Interculturalidad \\ HISULA \\ Licenciatura en Ciencias Sociales \\ Licenciatura en Psicopedagogía \\ Colombia
}

\section{RESUMEN}

Este estudio parte desde el objetivo de analizar la instrumentalización de menores en el contexto del conflicto armado colombiano, en la década de los setenta, enfatizando en la gravedad de los hechos y circunstancias debido a las repercusiones de la conducta como un crimen de guerra. Esto mediante una mirada crítica, donde aborda la violencia en la niñez en un periodo de tiempo establecido en las décadas de los 70, 80 y 90, con el reclutamiento de niños y niñas para la guerra. El propósito de este trabajo es analizar y reflexionar sobre la instrumentalización de menores en el contexto del conflicto armado. Para ello se tomó en cuenta experiencias documentales, informes y fuentes bibliográficas que se han articulado a las categorías de análisis tomadas para la investigación, en donde se da, desde una contribución a la memoria histórica del conflicto armado colombiano, situación decadente que involucra actores ajenos a la guerra donde la desolación de vidas incalculables ha sido utilizada con fines letales.

Por tanto, dentro de los resultados se encuentra que se genera un trastorno en sus conductas psicológicas y emocionales, sin ser ellos promotores de las mismas, de tal forma desde la mirada de autores se crea un análisis que permite identificar que las circunstancias vividas son trascendentales en estos niños y niñas, que viene a

\section{ENCUENTRO CON SEMILLEROS, APORTES Y REFLEXIONES}

Volumen 2 No 2 (2020) | ISSN 2711-4414 
explotar en consecuencias que lastiman no solo al sin sentido del conflicto armado colombiano, sino a todo el país.

Finalmente, estos sucesos se presentan en un país de alta desigualdad social donde los marginados son aquellos que se encuentran en las periferias, siendo vulnerables en este conflicto y lamentablemente sus vidas son distorsionadas convirtiéndose en víctimas y victimarios, en donde muchos de ellos mantuvieron esta experiencia cruel antes de su adolescencia.

PALABRAS CLAVE:

Conflicto armado, memoria, reclutamiento, violencia, menores

\section{INTRODUCCIÓN}

Esta investigación establece que el conflicto armado presenta una condición de complejidad inherente ya que puede comprenderse a través de las diferentes teorías que la respaldan, como la planteada por Anthony Guiddens y Acciones asociadas al poder, donde manifiesta que la estructura existe solo como "huellas mnémicas" o hábito que se actualiza en las acciones, y que estas, con las reglas y recursos que generan, son en gran parte responsables de la reproducción de los sistemas sociales'.

Por otra parte, Darío Antequera analiza cómo la memoria algunas veces es usada para legitimar políticas de ciertos grupos y que, por tanto, se plantea que la memoria debe construirse de manera colectiva, donde se tengan en cuenta los relatos de los diferentes agentes que estuvieron involucrados en las dinámicas que se presentan en el conflicto armado colombiano. En este sentido, se analiza los procesos que generó la guerra en los niños, niñas y adolescentes desde el autor del psicoanálisis, Freud, y Piaget con la teoría del desarrollo cognitivo, que presentan en sus teorías factores que permiten una interpretación sobre los sucesos que las personas afrontaron durante las etapas de su vida, por lo cual se realiza una analogía del conflicto armado en Colombia en la década de los 70, 80 y 90.

Considerando así para Ramírez,

sin lugar a duda, el reclutamiento es uno de los más graves y repudiables crímenes que se pueden cometer, porque suponen la negación de los derechos fundamentales de los más débiles, el desconocimiento de su dignidad humana, la imposibilidad de su derecho a decidir,

\footnotetext{
${ }^{1}$ Cambiasso, M.(2011). La teoría de la estructuración de Anthony Giddens: un ensayo crítico. 2011 ENCUENTRO CON SEMILLEROS, APORTES Y REFLEXIONES
} 
en definitiva, la supresión de toda manifestación de libertad. En el caso colombiano, el reclutamiento de niños y niñas se ha presentado al interior tanto de las organizaciones guerrilleras, paramilitares, como de las bandas criminales organizadas, las cuales han sido protagonistas de un conflicto armado que afecta al país desde hace más de cuatro décadas. En este escenario complejo y beligerante, los menores no solo resultan siendo víctimas del crimen del reclutamiento, sino que además pueden ser considerados victimarios de delitos cometidos con ocasión del conflicto, bien sea como autores o partícipes de delitos relacionados con la violación de los derechos humanos y del Derecho Internacional Humanitario, o por la comisión de delitos comunes. ${ }^{2}$

\section{MÉTODO}

Se consideró apropiado tomar como metodología la investigación cualitativa, que, desde su aplicación en las primeras consideraciones en la indagación de las temáticas abarcadas, se puede forjar múltiples herramientas metodológicas que promuevan la aprehensión y compresión de fenómenos psicológicos y sociales, lo que proporciona el análisis de diversas realidades, que en el transcurrir del tiempo construyen paulatinamente las personas en sus narrativas, desde historias, concepciones y reflexiones, gracias en gran medida a la rigurosidad metodológica que se maneja, al igual y no menos importante al aspecto ético que va de la mano en el transcurrir del trabajo con sus diversas técnicas (recolección y análisis de datos), que extienda de manera amplia las destrezas para manejar el tema de forma sensible, reflexiva y con una atención centrada en las narraciones de una guerra sin edad, expuestas en el informe nacional de reclutamiento ${ }^{3}$.

Esta línea metodológica permite comprender la raíz de ser víctima y convertirse en victimario, dado que se vuelve la niña y el niño reclutado en la víctima central, ya sea voluntario o forzado, vulnerando los derechos humanos de la infancia, y es en medio de sus actividades cotidianas como infante reclutado que inicia siendo una víctima por las violaciones y afectaciones psicológicas y emocionales que viven directa e indirectamente y, como lo mencionan las fuentes documentales tomadas de las experiencias de niños y niñas en la guerra, los alimentan con odio, dolor, venganza por medio de la represión de sus emociones que más adelante vienen a salir de la peor manera, y son estas víctimas las que se vuelven victimarios de la guerra, condicionados para enfrentar una violencia desgarradora, en donde Colombia desconoce el origen del victimario y los factores que incidieron su conducta, y es allí que se hace necesario en medio de los instrumentos de análisis el investigar desde un inicio que se relaciona con un final continuo, la guerra tergiversada y manipulada que deja en el olvido

\footnotetext{
${ }^{2}$ Ramírez. El Reclutamiento de menores en el conflicto armado colombiano. Aproximación al crimen de guerra. 2010.

${ }^{3}$ Una guerra sin edad, Informe nacional de reclutamiento y utilización de niños, niñas y adolescentes en el conflicto armado colombiano. 2017
}

\section{ENCUENTRO CON SEMILLEROS, APORTES Y REFLEXIONES}


la médula central de este trabajo como es la instrumentalización de la infancia para la guerra (DazaOrozco,2019).

\section{RESULTADOS}

Se presenta a continuación el desarrollo teórico del estudio, donde es importante identificar la construcción de un referente conceptual para generar un análisis del conflicto armado en Colombia ${ }^{4}$, que ha involucrado de forma directa a los niños, niñas y adolescentes para ser un instrumento de guerra por medio de su reclutamiento; al respecto se realiza un enfoque en tres conceptos que permitan contribuir al estudio en mención, para ello se establecerá un contenido sobre las acciones asociadas al poder, desde el autor Anthony Gidens, las memorias históricas como relato emblemático a partir de la mirada de José Antequera y finalmente el proceso mental en la memoria infantil a través de la visión de Freud, teniendo como punto de referencia el Centro Nacional de Memoria Histórica y su Informe nacional de reclutamiento y utilización de niños, niñas y adolescentes en el conflicto armado colombiano, en el que "Propone una mirada exhaustiva del fenómeno del reclutamiento y utilización de niños, niñas y adolescentes por parte de grupos armados en Colombia." ${ }^{5}$

Una guerra que destruyó sonrisas y cambió sus sueños por maldad, teniendo presente que la mayoría de ellos tenía difíciles condiciones económicas y familiares, en donde el ofrecimiento de otra forma de vida los llevó a enfrentar una cruel realidad social, porque las oportunidades son pocas y cada vez las limitaciones los llevaron a ser reclutados para la guerra desde los tres tipos: voluntario, forzoso y obligatorio, y cada normatividad externa e interna, conforme a las leyes desde el sistema de protección de los derechos humanos que se custodia en discursos y los niños se mantienen desprotegidos, de tal forma comienzan una vida en armas, porque su presente carecía de integridad y nadie salvaguardaba eso; se han convertido en armas donde el Estado no tuvo presencia y la visión del mundo se basó en órdenes de muerte, de tal manera se presenta los siguientes teóricos para amplificar el tema desde el estudio realizado.

\section{ACCIONES ASOCIADAS AL PODER}

\footnotetext{
${ }^{4}$ El reclutamiento de menores en Colombia constituye un crimen de guerra contenido en la legislación nacional y en el Estatuto de la Corte Penal Internacional, de innegable gravedad por la naturaleza de los derechos que resultan afectados con su comisión-

${ }^{5}$ (Centro Nacional de memoria historica 2017, 16)
}

\section{ENCUENTRO CON SEMILLEROS, APORTES Y REFLEXIONES}


Se toma en cuenta en el estudio La teoría de la estructuración de Giddens, ${ }^{6}$ que ofrece un camino para entender ejes fundamentales de una teoría del poder. En tanto que, en palabras de él, su planteamiento de que la estructura existe solo como "huellas mnémicas"”

o hábitos que se actualizan en las acciones, y que estas, con las reglas y recursos que generan son en gran parte responsables de la reproducción de los sistemas sociales; o dicho de otro modo, la afirmación de que las propiedades estructurales de los sistemas sociales existen solamente en tanto en cuanto las formas de conducta social se reproducen crónicamente a través del tiempo y el espacio, sale al paso del problema que se le presenta a Foucault o a los autores de corte marxista a la hora de explicar la acción humana como libre, pues esto cuesta de encajar con el presupuesto de que la estructura es determinante. Con Giddens el problema se resuelve de una manera muy simple: las estructuras sociales consisten en la rutinización de unas prácticas que se perpetúan por un tiempo. Las estructuras no son nada fuera de estas prácticas, y a la inversa: nuestros hábitos cotidianos son la principal fuerza que sostiene y conforma las estructuras. ${ }^{8}$

Foucault comparte también la idea de que son las prácticas sociales ${ }^{9}$ las que, perpetuándose en un espacio y un tiempo, están en el fundamento de la constitución tanto del objeto como del sujeto social, sin embargo, se siente como un "bache", como una dicotomía no resuelta en la intersección entre decir y hacer, o entre el discurso y la acción, tal como lo piensa este autor. Para salvar este "bache", Giddens introduce el concepto de conciencia práctica ${ }^{10}$. Por ello entiende "lo que los actores tácitamente saben sobre cómo manejarse en los distintos contextos de la vida social sin ser capaces de darles una expresión discursiva directa". Es tanto el concepto de rutinización como el de conciencia práctica pueden ser bien importantes para la teoría social. ${ }^{11}$ Como señala Giddens, "sería un error suponer que los componentes no discursivos de la conciencia son necesariamente más difíciles de estudiar empíricamente que los discursivos, a pesar de que por definición los propios agentes no puedan comentar directamente sobre ellos".

\section{MEMORIA HISTORIAS COMO RELATO EMBLEMÁTICO}

Darío Antequera afirma que la memoria en algunos casos es usada por sectores políticos para transmitir un relato en el cual se glorifica y se legitima cierto tipo de acciones en pro de la defensa de interés comunes, como

\footnotetext{
${ }^{6}$ Giddens, La constitución de la Sociedad (Amorrortu, Donostia, 1996) 61

${ }^{7}$ Giddens, La constitución de la Sociedad (Amorrortu, Donostia, 1996) 61

${ }^{8}$ Mussi, La Teoría de la estructuración en Giddens y su silencio. 2012

${ }^{9}$ Prácticas sociales y constitución de experiencia en Hegel y Foucault. 2019

${ }^{10}$ Mussi, La Teoría de la estructuración en Giddens y su silencio. 2012

${ }^{11}$ García, La teoría de la estructuración y su observación desde la acción: los límites del análisis.
} ENCUENTRO CON SEMI LLEROS, APORTES Y REFLEXIONES 
pueden ser la democracia y la defensa de los derechos humanos ${ }^{12}$; pero que deja de lado el relato de quienes han sido sometidos. En otras palabras, la memoria histórica solo enaltece los vencedores y deja de lado a quienes fueron víctimas de dicho sometimiento.

Por tal motivo Darío Antequera plantea que es necesario que la memoria histórica reconozca a quienes han sido vulnerados por el conflicto, un reconocimiento que debe hacer la sociedad, pues este reconocimiento se constituye como un derecho ${ }^{13}$, donde lo acontecido con las víctimas se vincule con la historia contada por el vencedor, de esta manera, el concepto de memoria histórica se define como "concepto de la recuperación, de la capacidad de articulación, de comprensión, de enfrentamiento del miedo, y demás efectos buscados con el sometimiento ${ }^{14 "}$.

Lo anterior no a partir de conmemoración de fechas, ni la exaltación de héroes y de sitios emblemáticos, sino a través de la narrativa de carácter general, de un periodo específico, que vincule las víctimas y que trascienda en la sociedad ${ }^{15}$, para así reflexionar, establecer responsabilidades, rescatar valores y evitar su repetición. Lo anterior debe ser aceptado de manera hegemónica por la sociedad, para de esta manera poder convertirse en lo que se categoriza como "memoria emblemática”"16, donde no sea impuesta desde algún sector político, sino desde un relato compartido por todos, como lo plantea Darío Antequera. ${ }^{17}$

Para el caso colombiano, se observa que en este gobierno se ha intentado tergiversar la memoria histórica, en el momento en que se impide el reconocimiento de las víctimas, y se siguen reproduciendo prácticas atroces como el asesinato de líderes sociales, además de la negación por parte del director de memoria histórica de Colombia de la existencia del conflicto armado en el país, al catalogar a los grupos insurgentes como bandoleros y terroristas; esto entre otros aspectos desembocan en que la construcción de memoria histórica del conflicto en Colombia se vea entorpecida por un sector político que quiere tergiversar la verdad y no reconocer las victimas afectadas por el conflicto.

PROCESO MENTAL, MEMORIA INFANTIL

\footnotetext{
${ }^{12}$ (Antequera, 2011, p.46)

${ }^{13}$ (Antequera, 2011, p.36)

${ }^{14}$ (Antequera, 2011, p.37)

${ }^{15}$ (Antequera, 2011, p.37)

${ }^{16}$ (Antequera, 2011, p.38)

${ }^{17}$ (Antequera, 2011, p.38)
}

ENCUENTRO CON SEMILLEROS, APORTES Y REFLEXIONES

Volumen 2 No 2 (2020) | ISSN 2711-4414 
Las actividades que realizan los menores cuando son reclutados afectan de múltiples maneras su salud física y mental, generada desde las vivencias personales que implican los actos violentos, donde ellos son los actores involucrados siendo cada uno de ellos un instrumento de terror mediante las acciones que se evidencian en un estudio del reclutamiento de menores en el conflicto armado colombiano ${ }^{18}$, donde hace mención sobre los deberes que realizan los niños y niñas: "Se les obliga a participar de ejecuciones sumarias, torturas, asesinatos, secuestros, ataques a civiles, instalación de minas antipersonas, entre otros"; ;9 al respecto, las conductas que se adquieren durante su proceso en los grupos crean las experiencias dolorosas, desde las normas disciplinarias, que afectan las psiquis de los menores durante su proceso de crecimiento hasta la vida adulta.

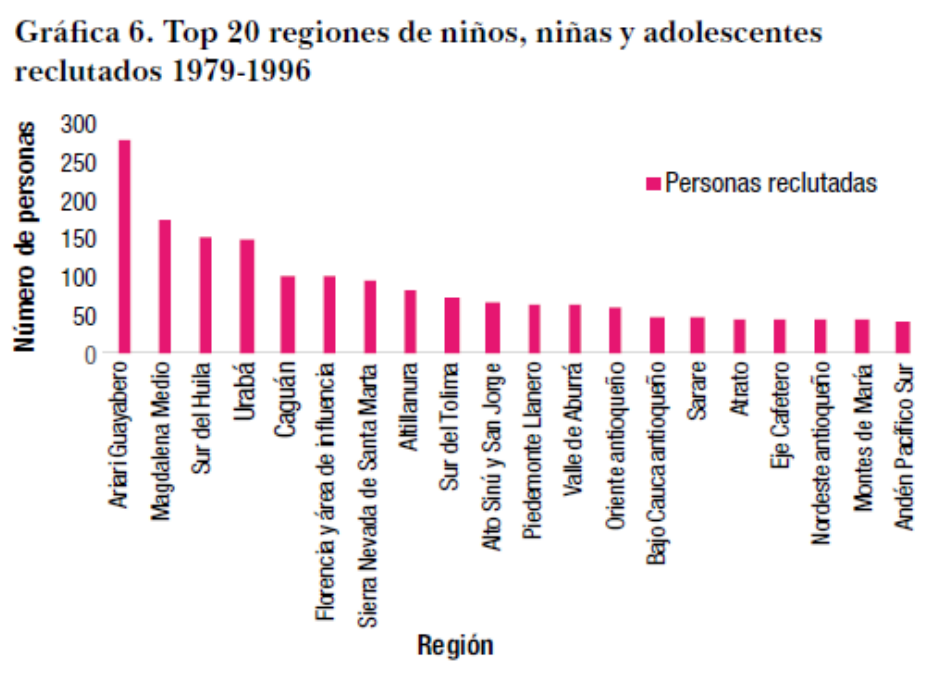

Fuente: Elaboración propia $\mathrm{CNMH}$, 2017, a partir de la Base de datos de reclutamiento y utilización, OMC, fecha de corte: 29/04/2017.

En la gráfica se observa el informe de Una guerra sin edad, que presenta la incorporación de menores a las guerrillas por región, en las décadas del siglo XX de mayor reclutamiento en Colombia durante el conflicto, los 70, 80 y 90. Estos niños, niñas y adolescentes, en el momento de ser parte de las filas comenzaron con una acumulación de recuerdos violentos en sus vivencias personales que serán repercutidos en su vida adulta, de tal manera, la parte emocional es fundamental en la memoria infantil; en este sentido, el informe del CNMH recopila algunos relatos de los cuales se tomaron tres para este análisis:

\footnotetext{
${ }^{18}$ Ibíd.

${ }^{19}$ (Ramirez Barbosa 2010, 29)
}

ENCUENTRO CON SEMILLEROS, APORTES Y REFLEXIONES

Volumen 2 No 2 (2020) | ISSN 2711-4414 
"Nos daban pólvora con guarapo. Nos servían, pero fuerte, de esos guarapos que lo emborrachan a uno, se lo servían a uno en un vasito, después destapaban la jiba y le echaban la pólvora ahí y se la tenía que tomar. La idea era del comandante, se lo daban a uno antes de salir a patrullar. Uno sentía rabia, como que eso era como pa' darle rabia a uno, sí me entiende, pa' cuando uno esté en el combate el objetivo es matar, matar, matar, ese es el objetivo (reclutado a los 14 años) ${ }^{20}$."

¡Ya se murió y ya!, no pasó nada, se entierra en cualquier lado. Se entierra donde quede y listo (...) Son muchísimos menores de edad muertos, por imprudencia a veces en los combates de pararse por lo nerviosos. Sea porque uno tiene que estar o sea porque tiene que avanzar en arrastre abajo sobre el piso. A veces uno se levanta por los nervios o que lo cojan a uno en una emboscada, todas esas cosas, la vida allá no vale prácticamente un peso (reclutado a los 13 años) ${ }^{21}$.

En la noche hay veces uno llegaba, hacía su campamento, uno se ponía a limpiar su fusil, limpiaba su fusil, después uno iba pa' la guardia, dos tres horas parado ahí en la guardia, por lo menos si uno se quedaba una noche dormido ahí en la guardia, lo mataban, lo mataban ¿sí pilla? Por lo menos uno está en la guardia y si usted dejaba el fusil, llegaba los mismos compañeros de uno y por hacerle la maldad le cogían el fusil a uno se lo escondían, le hacían maldad, lo mataban a uno e iban después por la familia. A mí me tocó mirar muchos amigos matar así solo por maldad. ${ }^{22}$

Estos son acontecimientos emocionales que marcan a los menores, y así tal cual lo mencionan algunos en sus relatos; “Uno sentía rabia, como que eso era como pa' darle rabia a uno, sí me entiende, pa' cuando uno esté en el combate el objetivo es matar, matar, matar, ese es el objetivo (reclutado a los 14 años)." Desde una mirada del psicoanálisis estos sucesos se van viviendo tal como lo menciona Freud, quedan en el inconsciente que tarde o temprano van a salir y de las peores formas. De tal manera, la psiquis de estos niños, niñas y adolescentes tiene una afectación directa, debido a que sus procesos de crecimiento y desarrollo -tanto físicos como mentales- se ven alterados; así mismo se debe tener en cuenta las invariantes funcionales de Jean Piaget, donde está la asimilación, que se puede comparar con el cambio de vida que se da en estos niños, niñas y adolescentes al momento de iniciar su vida en las filas; luego de ello viene la acomodación, cuando ellos entran a ser parte de esa vida, en relación con sus costumbres y hábitos.

\footnotetext{
${ }^{20}$ (Centro Nacional de memoria historica 2017)

${ }^{21}$ (Centro Nacional de memoria historica 2017)

${ }^{22}$ (Centro Nacional de memoria historica 2017)
}

\section{ENCUENTRO CON SEMILLEROS, APORTES Y REFLEXIONES}


En un sentido más amplio, se vivencia la adaptación, donde se ajustan a la vida que iniciaron y tienen que enfrentar la realidad que ya entra a ser parte de sus vidas. Este es un proceso que fuera de ser el incorrecto se ve reflejado en las alteraciones psicológicas que adquieren por el cambio de vida, permitiendo que el niño, niña y adolescente cambie su perspectiva de la sociedad y se adentre en la navegación de ideales que, aunque cercanos a su realidad, no permiten que haya un desarrollo total en relación con sus capacidades intelectuales y físicas.

Finalmente, el dar a conocer y concientizar es la forma sólida en que se logra mitigar la instrumentalización de la infancia en la guerra que, como se refleja en los estudios, desaparecen las vidas soñadas, las sonrisas inocentes, los juegos sanos y el respeto hacia el ser humano, que se logre una coherencia de pensamiento y acción, donde no se sea ajeno a una guerra nacional que ha invadido el corazón de Colombia.

\section{Intervención de modelo latinoamericano de la infancia}

Las áreas humanas, como las ciencias sociales y la psicopedagogía, permiten desarrollar y analizar un posible modelo latinoamericano con percepción conjunta en una educación donde los niños y niñas que han vivido la guerra o estén cercanos a ella puedan encontrar un desarrollo de sus habilidades en pro de su bienestar emocional, psicológico y físico por medio de la cultura visual (teniendo en cuenta la inclusión, se trata con personas que tengan una limitación visual, por medio de los otros sentidos). Lo que se busca con ello es, en primer lugar, desnaturalizar la guerra tanto para ellos como para sus familias a partir de la transformación de experiencias negativas de la violencia, en construcciones de su ser integral por medio de principios y valores reflejados en el arte que se estipule en todos los programas educativos a nivel nacional, y el generar conciencia no solo entre quienes han sido víctimas y victimarios, sino también en las personas externas que tienen una relación estrecha con el foco de estudio.

En ese sentido, Mirzoeff (2003) menciona que, "está claro que los estudios culturales y la cultura visual poseen su significado de cultura como un marco interpretativo". ${ }^{23}$ Esta cultura visual permite englobar las destrezas de niños, niñas y adolescentes, víctimas o victimarios de la guerra que puedan revindicar los sucesos lamentables que han tenido que vivir y transformarla educativamente, involucrando necesariamente las acciones cotidianas.

\section{DISCUSIÓN Y CONCLUSIÓN}

${ }^{23}$ MIrzoeff, N. Una introducción a la cultura (2013).

\section{ENCUENTRO CON SEMILLEROS, APORTES Y REFLEXIONES}

Volumen 2 No 2 (2020) | ISSN 2711-4414 
La investigación presentada destaca cómo la guerra afecta de manera irreparable las vidas de los niños y niñas del conflicto armado colombiano ${ }^{24}$, en las décadas de los 70,80 y 90, donde se contribuye en la memoria histórica de la violencia desde un enfoque psicológico en la infancia, esto para comprender los comportamientos que se generan y cómo estos hacen que la sociedad en general tergiverse la verdad y no reconozcan su proceso en la guerra, negándoles muchas veces la oportunidad de resocialización con la comunidad debido a sus experiencias, que de forma directa afectaron sus aspectos psicológicos y culturales.

La memoria histórica permite reconocer a los actores sociales que han incidido en el conflicto armado de Colombia dentro de varios momentos de la historia, a través de sus propias experiencias, vivencias en el proceso del reclutamiento que se da en temprana edad y las consecuencias del mismo; a su vez, la voz de estas personas permite mostrar el inmenso valor de la palabra que en varias ocasiones es marginalizada.

Finalmente, el conflicto armado en Colombia es un suceso que ha generado un sinfín de consecuencias desalentadoras para el país, en gran medida evidenciadas en la infancia que es en un porcentaje mayoritario la más afectada y es invisibilizada dentro de una memoria histórica que es ajena a la vida de los niños y niñas de Colombia. Esta investigación hace un llamado a no ser ajenos a estas vidas que en cierta manera fueron destruidas por ser un instrumento para la guerra. También es importante que se conozca las consecuencias internas del precio del conflicto armado colombiano.

\section{REFERENCIAS BIBLIOGRÁFICAS}

Antequera, J. (2011). Memoria histórica como relato emblemático. Consideraciones en medio de la emergencia de políticas de memoria en Colombia. Bogotá: Pontificia Universidad Javeriana. Facultad de Ciencias politicas y Relaciones internacionales; Maestría en Estudios políticos.

Arboccó, M. (2009). Piaget y Freud: acerca de la memoria infantil. Revista de Investigación en Psicología, (12), $207-215$

Cambiasso, M. (2011). La teoría de la estructuración de Anthony Giddens: un ensayo crítico. VI Jornadas de Jóvenes Investigadores. Instituto de Investigaciones Gino Germani, Facultad de Ciencias Sociales, Universidad de Buenos Aires, Argentina.

Centro Nacional de memoria histórica. (2017). Una guerra sin edad: un informe de reclutamiento y utilizacion de niños, niñas y adolescentes en el conflicto armado colombiano. Bogotá: Centro nacional de memoria histórica.

\footnotetext{
${ }^{24}$ Ibíd
}

\section{ENCUENTRO CON SEMILLEROS, APORTES Y REFLEXIONES}


Daza-Orozco, CE. (2019). Historia de la infancia en el cine colombiano. Bogotá: Institución Universitaria Politécnico Grancolombiano. Retrieved from http://alejandria.poligran.edu.co/handle/10823/1648

García, A. (2009). La teoría de la estructuración y su observación desde la acción: los límites del análisis. Estudios Sociológicos, 27(79), 31-61

Giddens, A. (1996). La constitucion de la sociedad. Amorrortu: Donostia.

Mirzoeff, N. (2013). Una Introducción a la Cultura.

Mussi, R. (2012). La Teoría de la Estructuración en Giddens y su Silencio (Tesis de maestría en Sociología.) Facultad latinoamericana de Ciencias sociales, Sede Argentina

Ramírez, P. (2010). El Reclutamiento de menores en el conflicto armado colombiano. Aproximación al crimen de guerra. Derecho Penal y Criminología, 31(9), 115-136. 